\title{
Sliding mode control of biogas production by anaerobic digestion with addition of acetate
}

\author{
Plamena Zlateva \\ Institute of Robotics, Bulgarian Academy of Sciences, Acad. G. Bonchev Str., Block 2, P.O.Box 79, Sofia 1113, Bulgaria
}

\begin{abstract}
Biogas production by anaerobic digestion with addition of acetate is considered. Sliding mode control for regulation of the biogas flow rate using the addition of acetate as a control action is proposed. The control design is carried out with direct use of nonlinear model and expert knowledge. Chattering phenomena are avoided by realizing the sliding mode with respect to the control input derivative. The state variables, external disturbance, process output and control input are varied in the known intervals. The performance of the designed sliding mode control is investigated by varying the process set point and the uncertain process parameter, which reflecting the influence of the external disturbance. The excellent performance of presented control is proved through simulation investigations in MATLAB using Simulink. .
\end{abstract}

\section{Introduction}

In the recent years, there is an increasing interest by society and business for the use of green energy $[1,2]$. In particular, it is necessary to note that biogas is one very useful source of green energy [3, 4]. It can be seen as a promising type of biofuel.

The biogas production is typically done by anaerobic digestion (oxygen-free environment) of various organic material with the help of special microorganisms (biomass) $[4,5]$. This process generally carried out in continuously stirred tank bioreactors (CSTR).

The biogas consists mainly of methane $\left(\mathrm{CH}_{4}\right)$ and carbon dioxide $\left(\mathrm{CO}_{2}\right)$, and may have small amounts of hydrogen sulphide $(\mathrm{H} 2 \mathrm{~S})$, hydrogen $\left(\mathrm{H}_{2}\right)$, and carbon monoxide (CO). The gases methane, hydrogen, and carbon monoxide $(\mathrm{CO})$ can be combusted or oxidized with oxygen [6]. Almost all forms of organic material can be used to produce biogas. However, waste water, manure, energy crops and organic industrial waste are the most common feedstocks [7].

It is important to emphasize that the biogas production process is very complex and may sometimes become very unstable. Investigations have shown that addition of stimulating substances (acetate or glucose) in appropriate concentrations allows to stabilize the process and to increase the biogas flow rate $[8,9]$.

The process of biogas production is usually described by a system from ordinary differential equations with uncetain parameters. For these reason it is needed the developing of sophisticated control algorithms based on nonlinear models [10-13].

It is known that the sliding mode control is effectively used in the stabilization of nonlinear and uncertain plants [14]. The guaranteed system invariance to parameter uncertainties and external disturbances is the main advantage of the sliding mode [15-17]. However the control signal is discontinuous in time, which leads to chattering phenomenon.

In practice, such control is hard to realize therefore various techniques for chattering attenuation are proposed (boundary layers, auxiliary input variable, fuzzy logic, input-dependent sliding surface).

The aim of this paper is to design and investigate sliding mode control of the biogas production (anaerobic digestion) with addition of acetate as a control action.

\section{Process model}

In this study, the three-stage biogas production process described by the following nonlinear system from five ordinary differential equations and one algebraic equation is considered. The proposed system has two input variables [9]:

$$
\begin{aligned}
& \frac{d S_{0}}{d t}=-D S_{0}-\beta X_{1} S_{0}+Y_{p} D_{1} S_{0 i n} \\
& \frac{d X_{1}}{d t}=X_{1} \mu_{1}\left(S_{1}\right)-D X_{1} \\
& \frac{d S_{1}}{d t}=-k_{1} X_{1} \mu_{1}\left(S_{1}\right)+\beta X_{1} S_{0}-D S_{1} \\
& \frac{d X_{2}}{d t}=X_{2} \mu_{2}\left(S_{2}\right)-D X_{2} \\
& \frac{d S_{2}}{d t}=-k_{2} X_{2} \mu_{2}\left(S_{2}\right)+k_{3} X_{1} \mu_{1}\left(S_{1}\right)+D_{2} S_{2 i n}-D S_{2} \\
& Q=k_{4} X_{2} \mu_{2}\left(S_{2}\right)
\end{aligned}
$$


In this system (mass balance model), equation (1) describes the hydrolysis stage of the diluted organics with concentration $S_{0 \text { in }}(\mathrm{g} / \mathrm{L})$; equation (2) - the growth and changes of the acidogenic bacteria, with concentration $X_{1}(\mathrm{~g} / \mathrm{L})$, consuming the appropriate substrate, with concentration $S_{1}(\mathrm{~g} / \mathrm{L})$.

The mass balance for this substrate is described by equation (3), where the first term reflects the consumption by the acidogenic bacteria, the second term reflects the substrate formed during the hydrolysis stage and the third one - the effluent flow rate of liquid. Equation (4) describes the growth and changes of the methane-producing (methanogenic) bacteria, with concentration $X_{2}(\mathrm{~g} / \mathrm{L})$, consuming acetate, with concentration $S_{2}(\mathrm{~g} / \mathrm{L})$.

The mass balance equation for acetate (5) has four terms in his right side. The first term reflects the consumption of acetate by the methanogenic bacteria, the second one the acetate formed as a result of the activity of acidogenic bacteria, the third one the direct addition of acetate, with concentration $S_{2 i n}(\mathrm{~g} / \mathrm{L})$, (a new control input) and the last one the acetate in the effluent liquid.

The algebraic equation (6) describes the formation of biogas with flow rate $Q$ in $\left(\right.$ day $\left.^{-1}\right)$.

The specific growth rate of the acidogenic bacteria $\mu_{1}\left(S_{1}\right) \quad$ day $^{-1}$ ) and the specific growth rate of the methanogenic bacteria $\mu_{2}\left(S_{2}\right)\left(\right.$ day $\left.^{-1}\right)$ are described by known, nonlinear and differentiable function of time.

In this model $k_{i}, i=1, \ldots, 4$ are positive coefficients; $D_{1}\left(\right.$ day $\left.^{-1}\right)$, is the dilution rate for the inlet soluble organics, with concentration $S_{0 i n} ; D_{2}\left(\right.$ day $\left.^{-1}\right)$ is the dilution rate for the acetate, with concentration $S_{2 i n}$ added into the bioreactor and $D=D_{1}+D_{2}$ is the total dilution rate.

Generally $S_{0 i n}$ is an unmeasurable perturbation, while $S_{2 i n}$ is a known constant or control input. In all cases the washout of microorganisms is undesirable, that is why changes of the total dilution rate $D=D_{1}+D_{2}$ and the perturbation $S_{0 \text { in }}$ are possible only in some admissible ranges (for fixed value of $S_{2 i n}$ ):

$$
0 \leq D \leq D^{\max } ; \quad S_{0 \text { in }}^{\min } \leq S_{0 \text { in }} \leq S_{0 \text { in }}^{\max }
$$

\section{Equations Problem statement}

In this study, it is assumed that $D_{1}$ and $D_{2}$ are constants; $S_{0 \text { in }}$ is the external disturbance, which is uncertain but varied in known bounds; $S_{2 i n}$ - the control input and $Q$ the output.

It is well known that the first acidogenic stage (1)-(3) is independent of the second methanogenic stage (4)-(5).

This allows us to simplify the dynamic model (1)-(6) for control design purpose obtaining a new model with the following dynamical part [18]:

$$
\begin{aligned}
& \frac{d X}{d t}=\alpha S X-D X \\
& \frac{d S}{d t}=-k_{2} \alpha S X-D S+D_{2} S_{i n}+A \\
& Q=k_{4} \alpha S X
\end{aligned}
$$

where the variable $A=k_{3} \mu_{1} X_{1} \quad A=k_{3} \mu_{1} X_{1}$ is considered as a process parameter reflecting the influence of the external disturbance $S_{0 i n}$. In this simplified model the new state variables are $X=X_{2}$ and $S=S_{2}$. The specific growth rate of the methanogenic bacteria is rewritten as $\mu_{2}=\alpha S$, where $\alpha$ is the specific reaction rate.

The following assumptions are considerate:

a1). The state variables - the biomass and substrate concentrations are positive, bounded and differentiable functions of time:

$$
0<X^{\min } \leq X(t) \leq X^{\max } \text { and } 0<S^{\min } \leq S(t) \leq S^{\min },
$$

where $X^{\min }, X^{\max }, S^{\min }$ and $S^{\max }$ are known constants;

a2). The biogas production rate is a positive, bounded and differentiable function of time

$$
0 \leq Q^{\min } \leq Q(t) \leq Q^{\max },
$$

where $Q^{\min }$ and $Q^{\max }$ are known constants;

a3). The process input is the influent substrate concentration $u(t)=S_{i n}(t)$. It is a differentiable function of time and varied in known boundaries:

$$
0<S^{\max }<S_{\text {in }}^{\min } \leq S_{\text {in }} \leq S_{\text {in }}^{\max },
$$

where $S_{i n}^{\min }$ and $S_{i n}^{\max }$ are known constants; $S^{\max }$ is the maximum admissible value of the substrate concentration $S$;

a4). The specific reaction rate is known, nonlinear and differentiable function of time, depending on the state variables and the kinetics coefficients:

$$
\alpha(t)=\alpha(X(t), S(t), p) \quad \text { or } \quad \alpha(t)=\alpha(S(t), p),
$$

where $p$ is a vector of the kinetic coefficients;

a5). On the basis of expert knowledge the variation interval of the process parameters $A$ is given as:

$$
0<A^{\min } \leq A \leq A^{\max },
$$

where $A^{\min }$ and $A^{\max }$ are known constants;

a6). The state variable - substrate concentration $S(t)$ is directly or indirectly measured.

a7). The process output - biogas production rate $y(t)=Q(t)$ is measured on-line.

In this paper, the problem is to design a sliding mode control for regulation of the biogas production rate. 


\section{Sliding model control design}

Suppose it is desired to stabilize the process output $y(t)=Q(t)$ to its set-point $g=Q^{*}$. Hence, the biogas production rate error is:

$$
e(t)=Q(t)-Q^{*}=y(t)-g,
$$

where $g$ is positive constant.

In order to design the sliding mode control, the system (8)-(10) is transformed into a second order differential equation of the form:

$$
\begin{gathered}
\frac{d^{2} Q}{d t^{2}}+\left[D+\frac{k_{2}}{k_{4}} \frac{\eta S+\alpha}{\alpha S} Q-\frac{\eta S+\alpha}{\alpha S} \frac{d S}{d t}-\alpha S\right] \frac{d Q}{d t}+ \\
+\left[\left(-\frac{d}{d t}\left(\frac{\eta S+\alpha}{\alpha S}\right)+D \frac{\eta S+\alpha}{\alpha S}\right) \frac{d S}{d t}+\right. \\
\left.+(\eta S+\alpha)\left(\frac{k_{2}}{k_{4}} Q+D S-A\right)\right] Q= \\
=D_{2} Q \frac{\eta S+\alpha}{\alpha S} \frac{d S_{\text {in }}}{d t}+D_{2}(\eta S+\alpha) S_{\text {in }},
\end{gathered}
$$

where $\eta=\frac{d \alpha}{d S}$ by reason of replacement

$$
\frac{d \alpha}{d t}=\eta \frac{d S}{d t}
$$

Substituting (16) into (17), the following differential equation of the biogas production rate error is obtained:

$$
\frac{d^{2} e(t)}{d t^{2}}+a_{1} \frac{d e(t)}{d t}+a_{0} e_{1}(t)=b_{1} \frac{d u(t)}{d t}+b_{0} u(t)-a_{0} g,
$$

where

$$
\begin{aligned}
& a_{1}=D+\frac{k_{2}}{k_{4}} \frac{\eta S+\alpha}{\alpha S} Q-\frac{\eta S+\alpha}{\alpha S} \frac{d S}{d t}-\alpha S \\
& a_{0}=\left[\left(-\frac{d}{d t}\left(\frac{\eta S+\alpha}{\alpha S}\right)+D \frac{\eta S+\alpha}{\alpha S}\right) \frac{d S}{d t}+\right. \\
& \left.\quad+(\eta S+\alpha)\left(\frac{k_{2}}{k_{4}} Q+D S-A\right)\right] ; \\
& b_{1}=D_{2} Q \frac{\eta S+\alpha}{\alpha S}>0 ; \quad b_{0}=D_{2}(\eta S+\alpha)
\end{aligned}
$$

According to the proposed transformation, the investigated methane fermentation process (8)-(10) is considered as plant with differentiated control input. To guarantee the invariance of the variable structure system in sliding mode to the parameter uncertainty and external disturbance, it is required that certain matching conditions should hold. Moreover, for control design aim, the model (18), is represented by the generalized observability canonical form [14]:

$$
\begin{aligned}
& \frac{d e_{1}(t)}{d t}=e_{2}(t) \\
& \frac{d e_{2}(t)}{d t}=-a_{1} e_{2}(t)-a_{0} e_{1}(t)+b_{1} \frac{d u(t)}{d t}+b_{0} u(t)+a_{0} g
\end{aligned}
$$

where $e_{1}(t)=e(t)$.

To avoid the chattering phenomenon and to obtain a smoothed input signal, the sliding mode is realized with respect to the control input derivative. On the basis of the variable structure system theory, the control law in sliding mode is designed as follows [16]:

$$
\frac{d u(t)}{d t}=\psi_{1} e_{1}(t)+\psi_{2} e_{2}(t)+\psi_{3} u(t)+\psi_{4} g
$$

where $\psi_{i}, \mathrm{i}=1, . ., 4$ are switching coefficients.

It is well known that the necessary and sufficient condition for existence and stability of a sliding mode on switching surface $\sigma(t)$ is given by following the inequality $[15,16]$ :

$$
\sigma(t) \frac{d \sigma(t)}{d t}<0, \text { for } \forall \mathrm{t} \in \mathrm{R}^{+} \text {and } \sigma(t) \neq 0 .
$$

In this study, the sliding surface $\sigma(t)$ is defined, as follows:

$$
\sigma(t)=c_{1} e_{1}(t)+c_{2} e_{2}(t),
$$

where $c_{1}$ and $c_{2}$ are positive constants.

Taking into account (19)-(21) and (23), the stability condition (22) is guaranteed, when the switching coefficients are selected as follows:

$$
\begin{array}{ll}
\psi_{1}=-\psi_{1}^{*} \operatorname{sgn}\left(e_{1}(t) \sigma(t)\right) ; & \psi_{2}=-\psi_{2}^{*} \operatorname{sgn}\left(e_{2}(t) \sigma(t)\right) ; \\
\psi_{3}=-\psi_{3}^{*} \operatorname{sgn}(u(t) \sigma(t)) ; & \psi_{4}=-\psi_{4}^{*} \operatorname{sgn}(g \sigma(t))
\end{array}
$$

where

$$
\begin{aligned}
& \psi_{1}^{*}>\sup _{t}\left[a b s\left(\frac{a_{0}}{b_{1}}\right)\right] ; \quad \psi_{2}^{*}>\sup _{t}\left[a b s\left(\frac{c_{2} a_{1}-c_{1}}{c_{2} b_{1}}\right)\right] ; \\
& \psi_{3}^{*}>\sup _{t}\left[a b s\left(\frac{b_{0}}{b_{1}}\right)\right] ; \quad \psi_{4}^{*}>\sup _{t}\left[a b s\left(\frac{a_{0}}{b_{1}}\right)\right] .
\end{aligned}
$$

The calculation of the constant values $\psi_{i}^{*}, i=1, \ldots, 4$ is reduced to solving the optimization problem taking into account the assumption a1)-a7) and the condition (20).

Therefore the control law (21) can be rewritten in the form:

$\frac{d u(t)}{d t}=-\left(\psi_{1}^{*}\left|e_{1}(t)\right|+\psi_{2}^{*}\left|e_{2}(t)\right|+\psi_{3}^{*}|u(t)|+\psi_{4}^{*}|g|\right) \operatorname{sgn}(\sigma(t))$

In ideal sliding dynamics

$$
\sigma(t)=c_{1} e_{1}(t)+c_{2} e_{2}(t)=0
$$

using the method of equivalent control [6], the model (20) is described by following differential equation: 


$$
\frac{d e_{1}(t)}{d t}=-\frac{c_{1}}{c_{2}} e_{1}(t)
$$

The equation (27) includes only previous given constants $c_{1}$ and $c_{2}$, which defines the dynamics of closed-loop system. Moreover, the system invariance in sliding mode to parameter uncertainties and external disturbance is evident.

\section{Simulation studies}

Simulation investigations in MATLAB using Simulink. are carried out to illustrate the effectiveness of the designed second order - sliding mode controller to stabilize biogas production processes (1)-(6) in presence of uncertainty and disturbances.

There are many complex functions to describe cell growth limited by a single substrate. However, Monodtype kinetics are widely used to express the specific reaction rate:

$$
\alpha(t)=\frac{\mu_{m}}{k_{s}+S(t)} .
$$

In this case, the kinetic coefficients vector is written as $p=\left(\mu_{m}, k_{s}\right)$, where $\mu_{m}$ is the maximum specific growth rate and $k_{s}$ is the saturation coefficient.

The following values of the parameters are adopted:

$$
\begin{aligned}
& \mu_{m}=0.25 ; \quad k_{s}=0.8 ; \quad k_{g}=8 ; \quad k=8.3 ; \\
& D_{2}=0.0125 ; \quad D=0.05 .
\end{aligned}
$$

The state variables, external disturbance, process output and control input are varied in the known intervals:

$$
\begin{array}{lll}
0.5 \leq X \leq 2.5 ; & 0.1 \leq S \leq 0.5 ; & 0.06 \leq A \leq 0.1 \\
0.1 \leq Q \leq 1.5 ; & 1 \leq S_{\text {in }} \leq 120 .
\end{array}
$$

On the basis of the conditions (25)-(26) and (28)-(30), the control law is obtained as follows:

$$
\frac{d u}{d t}=-\left(80\left|e_{1}\right|+150\left|e_{2}\right|+0.2|u|+80|g|\right) \operatorname{sgn}(\sigma),
$$

where the sliding surface is

$$
\sigma(t)=0.4 e_{1}(t)+1 e_{2}(t) .
$$

The performance of the designed sliding mode control is investigated by varying the process set point and the uncertain parameter $A$.

The simulation results obtained from the step changes of the set-point

$$
(g=0.6 \rightarrow 0.9 \rightarrow 0.6 \rightarrow 0.1)
$$

are presented in Fig. 1. (a, b, c).

The good behavior of the nonlinear process and the smooth control input are evident.

Simulation experiments where the process parameter $A$ is changed as

$$
A=0.082 \rightarrow 0.056 \rightarrow 0.082 \rightarrow 0.0984 \rightarrow 0.082
$$

are shown in Fig. 2. (a, b)
It is evident from figure that the system invariance to parameter uncertainty is guaranteed.
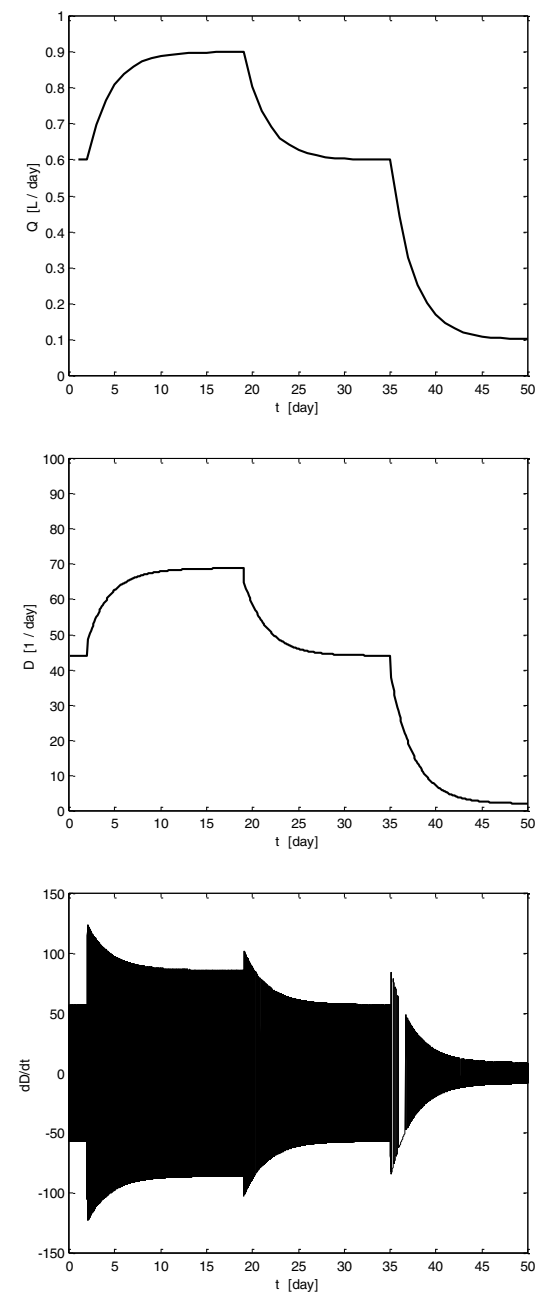

Fig. 1. Process behavior when the set-point is changed
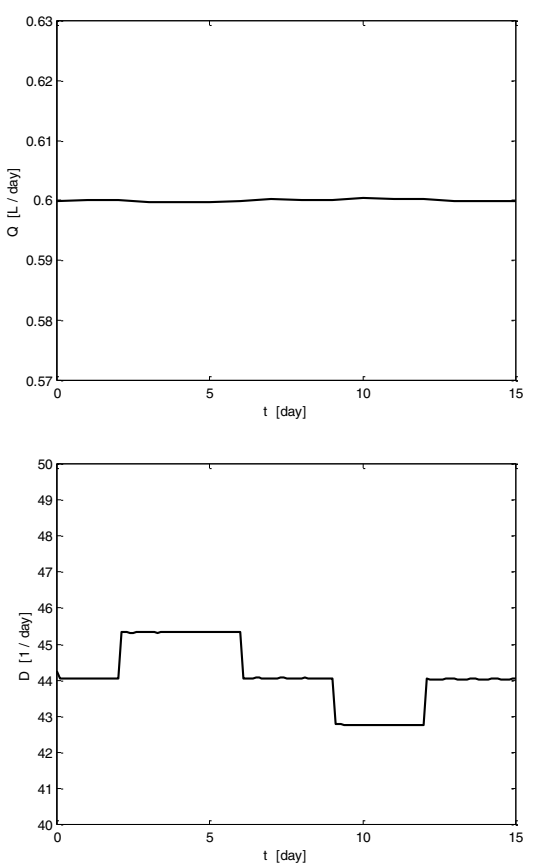

Fig.2. Process behavior when the parameter $A$ is changed 


\section{Conclusions}

The sliding mode control for biogas flow rate regulation with addition of acetate is proposed. The control design is carried out with direct use of nonlinear model of the biogas production by anaerobic digestion with addition of acetate and expert knowledge. Smooth control input is obtained. The state variables, external disturbance, process output and control input are varied in the known intervals. The simulation results prove good performance of the developed sliding mode control with respect to set point changes and external disturbances through simulation investigations in MATLAB using Simulink.

\section{References}

1. N. Scarlat, J.-F. Dallemand, Fernando Fahl, Renewable Energy, 129, Part A, 457-472 (2018)

2. REN21, Renewable Energy Policy Network for the 21st Century (2016)

3. F. van Foreest, Perspectives for Biogas in Europe, (Oxford Institute for Energy Studies, 2012)

4. A Wellinger, JD Murphy, D Baxter, The biogas handbook: science, production and applications (IEA Bioenergy, 2013)

5. S. Achinas, V. Achinas, G. J. W. Euverink, Engineering, 3 (3), 299-307 (2017)

6. Clarke-energy - A Kohler Company, Biogas \& Engines, (www.clarke-energy.com, 2011)
7. T. Al. Seadi, C. Lukehurst, Quality management of digestate from biogas plants used as fertiliser, (Task 37 - Energy from Biogas, IEA Bioenergy, 2012)

8. Deutsche Energie-Agentur GmbH (DENA), German Energy Agency Biogaspartner Project (https://www.biogaspartner.de/startseite, 2017)

9. B.D. Wellinger, J. Murphy, The Biogas Handbook. Science, Production and Applications (Woodhead Publishing 2013)

10. A. Ghouali, T. Sari, J. Harmand, Maximizing biogas production from the anaerobic, (HAL Id, hal.archives-ouvertes.fr, 2015)

11. N. Dimitrova, M. Krastanov, Int. J. Robust. Nonlinear, (wileyonlinelibrary.com, 2011)

12. Mazenc, F., J. Harmand, H. Mounier, NOLCOS (Toulouse, France, 2013)

13. I. Simeonov, E. Chorukova, S. Diop, N. Christov, Termotehnica 2, 8 (2014)

14. P. Zlateva, Bioprocess Engineering 16 (1), 383-387 (1997)

15. Utkin V. Sliding modes in optimization and control problems (Springer-Verlag, New York, 1992)

16. Y. Shtessel, C. Edwards, L. Fridman, A. Levant, Sliding mode control and observation (Springer, Basel, 2014)

17. L. Fridman, J.-P. Barbot, F. Plestan, Recent Trends in Sliding Mode Control (CPI Group Ltd, 2016)

18. M. Borisov, N. Dimitrova, I. Simeonov, IFACPapersOnLine 49-26, 231-238 (2016) 